\title{
A Refinement of Clinical Tumor Marker Monitoring: Why Not Use an Inverse Value of Doubling Time?
}

\author{
Gintaras Zaleskis $^{a}$ Paulius Bosasa, ${ }^{a}$ Albertas Ulys ${ }^{a}$ Daiva Dabkevičiene ${ }^{a}$ \\ Neringa Dobrovolskiene ${ }^{a}$ Bret Andrew Hudson ${ }^{c}$ Vita Pašukoniene ${ }^{a, d}$

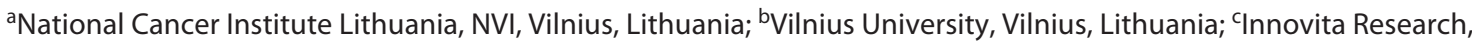 \\ Ltd., Vilnius, Lithuania; ${ }^{\mathrm{d}}$ Gediminas Technical University, Vilnius, Lithuania
}

\section{Highlights of the Study}

- Cancer recurrence is easier to spot referring to the kinetics of doubling time rather than tumor marker norms.

- An inverse value of tumor maker doubling time is a versatile parameter free from mathematical drawbacks.

- This study demonstrates that prostate-specific antigen kinetic parameter ePSA can predict biochemical recurrence postoperatively.

- The ePSA can be considered for implementation in daily clinical practice.

\section{Keywords}

Tumor markers - Cancer relapse - Prostate cancer · Prostatespecific antigen

\footnotetext{
Abstract

Objectives: The aim of this study was to compare prostatespecific antigen (PSA) kinetics - half-life time (HT), doubling time (DT), and elimination rate PSA (ePSA) in prostate cancer (PCa) monitoring. Implementation of ePSA in clinical practice could help simplify patient monitoring in the remission phase. Materials and Methods: A total of 49 PCa patients were examined by their PSA tests before prostatectomy and after 30 days, 91 days, and 24 months. Conventional PSA rate of change parameters (HT and DT) were compared to a new
}

clinically understandable ePSA parameter. Results: We observed that implementation of inverse value (ePSA) rather than HT or DT has distinct advantages: (1) values are valid when PSA is unchanged (ePSA equals zero), (2) the concept of ePSA can be easily understood, as it is a growth fraction, (3) ePSA fluctuates within a narrow range and is thus easy to interpret, and (4) there are no mathematical flaws (no positive skewing). Conclusion: Exploring ePSA norm as $\leq 0 \%$ could help spot biochemical recurrence in a timely manner. Primary health care providers tend to use an irrelevant PSA threshold, that is, $4.0 \mathrm{ng} / \mathrm{mL}$, in postoperative follow-up. The delayed referrals of patients in remission might be reduced if ePSA testing is adopted.

(C) 2021 The Author(s) Published by S. Karger AG, Basel karger@karger.com www.karger.com/mpp

Karger $\stackrel{\text { ' }}{5}$

GOPEN ACCESS
(C) 2021 The Author(s)

Published by S. Karger AG, Basel

This is an Open Access article licensed under the Creative Commons Attribution-NonCommercial-4.0 International License (CC BY-NC) (http://www.karger.com/Services/OpenAccessLicense), applicable to the online version of the article only. Usage and distribution for commercial purposes requires written permission.
Correspondence to:

Gintaras Zaleskis, gintaras.zaleskis@ nvi.lt 


\section{Introduction}

Surgical removal of tumor may result in decline in levels of tumor markers (TMs). TM kinetics in remission are often ignored by primary health care providers (PHCPs) when values oscillate in the range below norm. This approach might delay the recognition of recurrence $[1,2]$. A rise in TMs may precede the clinical appearance of recurrence by $>6-17$ months $[1,3]$. The currently explored criteria, namely, doubling time (DT), can be used as an indicator of relapse [4]. However, half-life time (HT)/DT criteria are not convenient in clinical practice for several reasons: (i) HT/DT values are undefined when TM is stable (dividing by 0 ), (ii) large and highly variable values are hard to interpret, and (iii) mathematical drawbacks, distribution asymmetry is positively skewed [5].

We proposed to study the use of an inverse value of HT/DT in TM monitoring. The concept is identical to the pharmacokinetic elimination rate constant, showing an inverse value of the HT of the amount of drug injected. We refer to this as an elimination rate TM (eTM); this can be defined as a "growth fraction" or "elimination fraction," and eTM is mathematically valid when there is no change in TMs (eTM equals 0 ). We hypothesized that monitoring eTM could be helpful in the detection of early recurrence when TM is increasing within the normal range.

Delayed detection of relapse sometimes occurs during primary care follow-up. An example could be a radical prostatectomy (RP) where prostate-specific antigen (PSA) levels should become undetectable following tumor removal [6]. However, PHCPs tend to habitually use a PSA threshold which is too high $(4.0 \mathrm{ng} / \mathrm{mL})$ to monitor patients postoperatively [2]. Biochemical recurrence (BCR) of prostate cancer $(\mathrm{PCa})$ must be spotted significantly earlier when $\mathrm{PSA}=0.2 \mathrm{ng} / \mathrm{mL}$ [7]. It may take years to accomplish a transition of care from PHCPs to oncologists. The risk of delayed referrals and missed opportunities might be linked to the PSA norm embedded in laboratory reports $(<4 \mathrm{ng} / \mathrm{mL})$ which is unrelated to postoperative norms (undetectable).

We evaluated the clinical applicability of eTM (ePSA) compared to its inverse equivalent (HT/DT) in PCa patients. The specific ePSA patterns observed postoperatively between days 30 and 91 indicated a BCR of PSA.

\section{Materials and Methods}

Calculation of ePSA:

$$
\mathrm{ePSA}=\frac{100}{\mathrm{HT}}=\frac{100 \times \ln \left(\frac{N}{M}\right)}{\ln 2 \times(\Delta t)},
$$

Refinement of Clinical Tumor Marker Monitoring where $N=$ current PSA and $M=$ previous PSA. ePSA values $<0$ appear if PSA is decreasing and $>0$ if it is increasing. $\Delta t=$ time interval, expressed in days between two separate PSA measurements. The ePSA metric is "percent/day" reflecting growth or elimination fraction. In this communication, we use $\%$ metric to simplify concept. PSA value adjustment to a specific sampling day (e.g., day 30) was done using the following equation:

$\mathrm{PSA}_{\text {day } 30}=\mathrm{PSA}_{\mathrm{M}} \times 2^{(M-30) / \mathrm{HT}}$
$\mathrm{PSA}_{\text {day } 91}=\mathrm{PSA}_{\mathrm{M}} \times 2^{(M-91) / \mathrm{HT}}$,

where $\mathrm{PSA}_{\text {day } 30}=\mathrm{PSA}$ value as if it was measured on day 30; PSA $_{M}=$ actual PSA value at $M$ representing a "1-month" time point; $M=$ number of days from RP to PSA measurement; $\mathrm{HT}=$ half-life time of PSA from RP to $M$ time point. PSA $A_{\text {day } 91}$ was used to adjust values to represent a "3-month" time point. ePSA calculations with multiple PSA values (Fig. 1c) can be applied exploring nomograms available for DT calculation [8].

\section{Patients and Tests}

The study was approved by an Institutional Review Board. Informed consent was obtained from all patients. A total of 49 previously untreated patients with a histologically confirmed diagnosis of adenocarcinoma in the prostate received RP at our institute from November 2017 to May 2018. The follow-up PSA data were obtained using an ultrasensitive PSA assay (Cobas e411; Roche Diagnostics, Risch-Rotkreuz, Switzerland). All patients enrolled exhibited normal ranges of liver enzymes, bilirubin, and creatinine during the follow-up. No blood transfusions were done during the period of PSA measurement. Clinicopathological characteristics of the study participants were as follows: age - mean $62.5 \pm 6.2$ years, BMI - mean $25.1 \pm 2.84 \mathrm{~kg} / \mathrm{m}^{2}$, and Karnofsky status $\geq 70 \%$. Adenocarcinoma of the prostate was confirmed based on preoperative biopsy findings. All patients displayed a negative CT scan/MRI and bone scan for metastatic PCa. Conventional open radical retropubic RP was performed extraperitoneally in a retrograde fashion. The laparoscopic extraperitoneal RP was performed using a 5-trocar technique. Total intravenous anesthesia with propofol without sevoflurane or opioid was applied to all study participants. None of the patients received radiotherapy or androgen deprivation therapy within 6 months after the surgery. The post-RP follow-up for each patient included a PSA measurement every 3 months. BCR was defined as a confirmed increase in PSA above $0.2 \mathrm{ng} / \mathrm{mL}$.

\section{Statistical Analysis}

IBM-SPSS Statistics 21 (SPSS, Inc., Chicago, IL, USA) was used for data analysis. Binary logistic regression and receiver operating characteristic were performed to determine the effectiveness of ePSA in predicting BCR. $p$ values $<0.05$ were considered statistically significant.

\section{Results}

Preoperative PSA was found as mean $9.0 \pm 7.3 \mathrm{ng} / \mathrm{mL}$ (median $-8.2 \mathrm{ng} / \mathrm{mL}$ ); pathological status: 35 patients (pT2) and 14 patients (pT3); Gleason score at surgery was found as follows: 12 patients $\rightarrow(3+3), 30$ patients $\rightarrow(3$ 

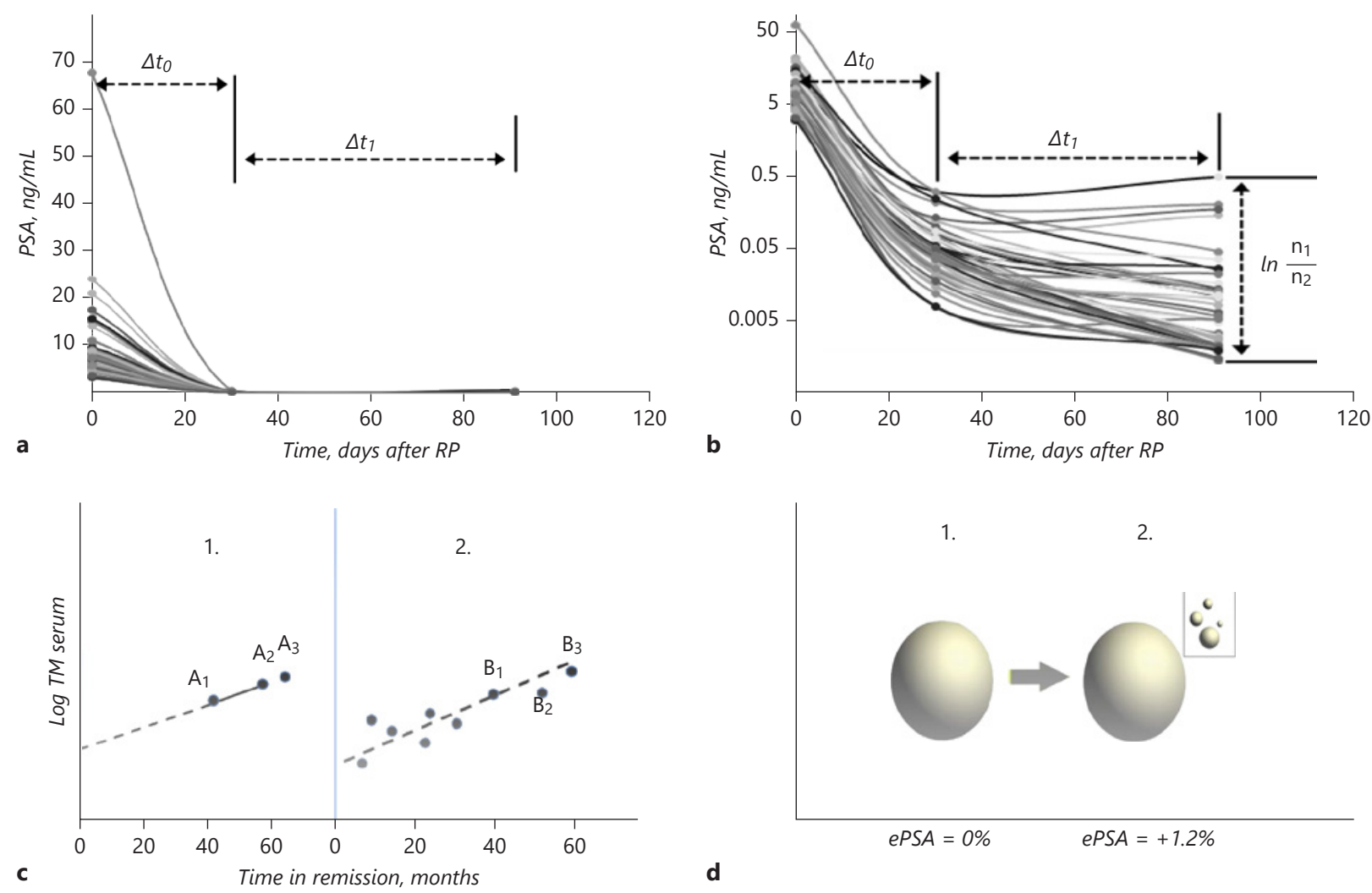

\begin{tabular}{|c|c|c|}
\hline $\begin{array}{l}\text { Example: the same PSA } \\
\text { value (with and without } \\
\text { ePSA) determined three } \\
\text { years following surgery }\end{array}$ & $\begin{array}{c}\text { Decision making option once ePSA is available; } \\
\text { the rough estimation by adding ePSA value to } \\
\text { PSA (e.g,: } 0.18+0.01=0.19 \text { in one day, or } 0.20 \\
\text { in two forthcoming days); } 1 \%=0.01 .\end{array}$ & $\begin{array}{l}\text { Current practice based merely } \\
\text { on criteria of biochemical } \\
\text { recurrence: "PSA cut-off level } \\
\text { is } 0.2 \mathrm{ng} / \mathrm{ml} \text { " }\end{array}$ \\
\hline $0.18 \mathrm{ng} / \mathrm{ml}$ & na & \\
\hline $\begin{array}{c}0.18 \mathrm{ng} / \mathrm{ml} \\
\text { ePSA }=+1.0 \% \\
(\text { "aggressive") }\end{array}$ & $\begin{array}{c}\text { PSA most probably will exceed cut-off level of } \\
0.2 \mathrm{ng} / \mathrm{ml} \text { in several days. Schedule next PSA } \\
\text { test in a week? }\end{array}$ & $\begin{array}{l}\text { Schedule next appointment } \\
\text { and PSA testing after } 6 \\
\text { months }\end{array}$ \\
\hline $\begin{array}{c}0.18 \mathrm{ng} / \mathrm{ml} \\
\text { ePSA }=-0.03 \% \text { ("stable") }\end{array}$ & $\begin{array}{c}\text { PSA value most probably will remain the same } \\
\text { after } 6 \text { months; }\end{array}$ & \\
\hline
\end{tabular}

Fig. 1. The value of logarithm in evaluating TM development. The PSA data (preoperative, day 30 and day 91) of the same cohort are shown using a linear scale (a) and log scale (b). Any claim in colloquial clinical language saying TM "percent increase" (decrease) does not reflect the evolution of TM within a given time interval. Importantly, these claims rarely indicate a precise time interval between two separate TMs. An exact time interval and log TM ratio are two basic components which can describe the "speed" of progress or decay of TM. Trying to squeeze a TM ratio into linear concept of increase [11] results in a discussion on which of the 7 patterns is the most informative. Any new confirmative TM test should be evaluated if it fits into the pattern of the previous two, in a log TM scale only (c). The exact timing of planned A3 point determination is not that important, provided the TM is tested with the same instrument. The availability of multiple TM points is advantageous (c). Calculation of an eTM with multiple points for each patient is available using nomograms with minimal conversion of DT into eTM [8]. A hospital lab can produce automatic reports for eTM if previous data for the same patient are available. A clinician can easily adopt the simplicity of growth fraction concept and can assume that a $\sim 1 \%$ daily increase in TM will result in a roughly $30 \%$ increase of TM value within a month (1\% per day results roughly in $30 \%$ per month) (d). TM, tumor marker; PSA, prostate-specific antigen; eTM, elimination rate TM; DT, doubling time; $\mathrm{RP}$, radical prostatectomy; ePSA, elimination rate PSA. 
$+4), 6$ patients $\rightarrow(4+3)$, and 1 patient $\rightarrow(4+4)$. Positive surgical margins were found in 6 patients. Surgical procedures applied were: open RP (18 patients) and laparoscopic RP (31 patients). The 1-month blood sampling day ranged from day 27 to 38 (mean 31.1; median 30) after RP. The mean PSA-HL was 4.1 days, corresponding to an ePSA $=-24.6 \%$. After conforming PSA to an exact 30-day time point, the adjustments ranged from +0.008 to -0.326 $\mathrm{ng} / \mathrm{ml}$. A significant slowing in PSA decline was observable during the 1- to 3-month interval (Fig. 1b). Mean PSA-HL at 3 months was $+21.4 \mathrm{~d}$, ranging from -238.7 to $+374.6 \mathrm{~d}$, and a median of $+19.3 \mathrm{~d}$. The calculated mean ePSA value for the same period was $-4.67 \%$. A logarithmic scale of postoperative PSA is necessary for PSA pattern visualization (Fig. 1a, b). Four patients (8\%) showed a bounce in PSA with values higher at 3 months as compared to their 1-month values. Patients exhibiting a bounce in PSA (positive ePSA) or slowing of PSA decline (ePSA $>-2 \%$ during the 1 - to 3 -month interval) were prone to BCR within 24 months after RP: odds ratio $(\mathrm{OR})=1.5, p=0.006,99 \% \mathrm{CI}(1.136 ; 2.139)$.

\section{Discussion}

This study was aimed at reviewing the complexity of methods describing TM kinetics and at proposing a solution to the complexity of implementing the inverse HT/DT parameter. The presence of wide ranging and large numerical HT values was reported in therapeutic docetaxel monitoring [9]. For instance, a very slow response to docetaxel from this study $(+12,734$ days HT) would instead be a reasonable ePSA of $-0.01 \%$ (percent PSA decrease per day). The group of patients examined during the postoperative period in our study was ascribed HT/DT or ePSA criteria. The data were conveyed to PHCPs after they were familiarized with the "growth fraction" concept (Fig. 1d) of ePSA. For instance, a patient's PSA before RP was $9.56 \mathrm{ng} / \mathrm{mL}$. PSA levels in this patient were $0.365 \mathrm{ng} / \mathrm{mL}$ at 30 days following RP and $0.380 \mathrm{ng} / \mathrm{mL}$ at 91 days. While a stabilized PSA in the interval of 30-91 days postoperatively indicates a potential problem, the numerical value of this event and the clinical importance of the PSA oscillations are not available to clinicians in their daily routine. If a clinician is provided with a number as complicated as "minus 1,084.1 days HT," it is quite difficult to comprehend. Actually, this HT approach is not set as an adopted principle of current medical practice. Instead, we could offer to the clinician the value of $0.1 \%$ ePSA

Refinement of Clinical Tumor Marker Monitoring derived from the same patient's PSA data. This format would mean that the patient's PSA would increase by $0.1 \%$ or roughly $0.0004 \mathrm{ng} / \mathrm{mL}$ each day. This information is easy to understand, and it can help establish an approximate BCR time in variable clinical situations (Fig. 1). In fact, up to $67 \%$ of PHCPs might not be aware that postoperative PSA norm is categorized as "undetectable" [10]. A norm ePSA of "0 or less" might help PHCPs to spot a pattern of positive ePSA and BCR in a timely fashion. Several methods have been advocated to elucidate the relapse pattern of TM above and below normal range $[1,3,7,11]$. eTM has the advantage that there is no time restriction for testing (in theory, retesting can be done the next week); furthermore, its value is calculated from the ratio of two separate TM values in natural logarithm. This is overlooked when clinicians evaluate a TM increase or decrease based on a simple, nonlogarithmic ratio of two separate TMs.

The indirect evidence of the TM value pattern was demonstrated in a group of healthy women who were later diagnosed with ovarian cancer [12]. Serum Ca-125 testing revealed that a gradual and persistent increase in normal range was noticeable 3 years preceding the diagnosis. In fact, much of the research on early detection of cancer is focused exclusively on "elevated over the normal range" TM value rather than the "rate of change" of TM. This approach thus consumes an enormous amount of time and resources, only to come to a situation that has little hope of making progress.

For instance, 3 of 4 women with tumors had normal screening tests prior to diagnosis even though they were preselected according to genetic risk criteria, then were tested for Ca-125 and finally were assessed by transvaginal ultrasound [13]. Creating new screening strategies (or strategies to spot a recurrence) built on the rate of change rather than an elevated TM value might open additional opportunities in clinical oncology practice. Importantly, it is not the value itself but rather a steady kinetic pattern in the normal range that could be the earliest indicator of recurrence. The eTM could well serve as a tool to ascribe this in a simple numerical format.

\section{Conclusions}

We suggest that the ePSA parameter indicating the percent PSA change per day could be a useful adjunct to regular PSA reports. ePSA is free from drawbacks characteristic of HT and DT. The concept is easily understandable and could be considered for all TMs. 


\section{Statement of Ethics}

This study was approved by the Regional and Institutional Ethics Committee. It was conducted in accordance with the Declaration of Helsinki (2000) and the International Conference on Harmonization Guidelines for Good Clinical Practice. All patients provided written informed consent prior to enrollment.

\section{Conflict of Interest Statement}

The authors have nothing to declare.

\section{References}

1 Di Gioia D, Stieber P, Schmidt GP, Nagel D, Heinemann V, Baur-Melnyk A. Early detection of metastatic disease in asymptomatic breast cancer patients with whole-body imaging and defined tumour marker increase. Br J Cancer. 2015 Feb;112(5):809-18.

2 Chapman $\mathrm{CH}$, Caram MEV, Radhakrishnan A, Tsodikov A, Deville C, Burns J, et al. Association between PSA values and surveillance quality after prostate cancer surgery. Cancer Med. 2019, Dec;8(18):7903-12.

3 Wilder JL, Pavlik E, Straughn JM, Kirby T, Higgins RV, DePriest PD, et al. Clinical implications of a rising serum CA-125 within the normal range in patients with epithelial ovarian cancer: a preliminary investigation. Gynecol Oncol. 2003 May;89(2):233-5.

4 Freedland SJ, Humphreys EB, Mangold LA, Eisenberger M, Dorey FJ, Walsh PC, et al. Risk of prostate cancer-specific mortality following biochemical recurrence after radical prostatectomy. JAMA. 2005 Jul 27;294(4): 433-9.
5 Mehrara E, Forssell-Aronsson E, Ahlman H, Bernhardt P. Specific growth rate versus doubling time for quantitative characterization of tumor growth rate. Cancer Res. 2007 Apr 15; 67(8):3970-5.

6 Doherty AP, Bower M, Smith GL, Miano R, Mannion EM, Mitchell H, et al. Undetectable ultrasensitive PSA after radical prostatectomy for prostate cancer predicts relapse-free survival. Br J Cancer. 2000;83(11):1432-6.

7 Guidelines for radical treatment of locally-advanced disease (section 6.2.4.5.). European Association of Urology. EAU Guidelines Office, Arnhem, The Netherlands. http:// uroweb.org/guidelines/compilations-of-allguidelines/[Accessed 2020].

8 Prediction tools/Prostate cancer nomograms. Memorial Sloan Kettering Cancer Center. PSA doubling time. https://www.mskcc.org/ nomograms/prostate/psa_doubling_time [Accessed 2020].

9 Hanninen M, Venner P, North S. A rapid PSA half-life following docetaxel chemotherapy is associated with improved survival in hormone refractory prostate cancer. Can Urol Assoc J. 2009 Oct;3(5):369-74.
10 Yassaie O, McLaughlin B, Perera M, Manning T, Lawrentschuk N, Malcolm A. Primary care follow-up of radical prostatectomy patients: a regional New Zealand experience. Prostate Int. 2016 Dec;4(4):136-9.

11 Abu Hassan SO, Nielsen DL, Tuxen MK, Petersen PH, Sölétormos G. Performance of seven criteria to assess CA125 increments among ovarian cancer patients monitored during first-line chemotherapy and the post-therapy follow-up period. Future Sci OA. 2017 Jul 18; 3(3):FSO216. PMID: 28884012; PMCID; PMC5583662.

12 Anderson GL, McIntosh M, Wu L, Barnett M, Goodman G, Thorpe JD, et al. Assessing lead time of selected ovarian cancer biomarkers: a nested case-control study. J Natl Cancer Inst. 2010 Jan 6;102(1):26-38

13 Olivier RI, Lubsen-Brandsma MA, Verhoef S, van Beurden M. CA125 and transvaginal ultrasound monitoring in high-risk women cannot prevent the diagnosis of advanced ovarian cancer. Gynecol Oncol. 2006 Jan; 100(1):20-6. 